\title{
Gauge mediated supersymmetry breaking and string theory
}

\author{
S.P. de Alwis ${ }^{a}$ and Z. Lalak ${ }^{b}$ \\ ${ }^{a}$ Physics Department, University of Colorado, \\ Boulder, CO 80309, U.S.A. \\ ${ }^{b}$ Institute of Theoretical Physics, Faculty of Physics, University of Warsaw, \\ ul. Hoza 69, 00-681 Warsaw, Poland \\ E-mail: dealwiss@colorado.edu, zygmunt.lalak@fuw.edu.pl
}

ABSTRACT: We discuss the possibility of finding scenarios, within type IIB string theory compactified on Calabi-Yau orientifolds with fluxes, for realizing gauge mediated supersymmetry breaking. We find that while in principle such scenarios are not ruled out, in practice it is hard to get acceptable constructions, since typically, supersymmetry breaking cannot be separated from the stabilization of the light modulus.

Keywords: Supersymmetry Phenomenology, Strings and branes phenomenology

ARXIV EPRINT: 1011.5856 


\section{Contents}

1 Introduction 1

$\begin{array}{lll}2 & \text { KKLT model } & 4\end{array}$

$\begin{array}{lll}3 & \text { KKLT racetrack model in IIB } & 5\end{array}$

$\begin{array}{lll}4 & \text { LVS models } & 6\end{array}$

$\begin{array}{lll}5 & \text { Retrofitted models } & 7\end{array}$

6 Models with charged moduli — role of D-terms 4

$\begin{array}{lll}7 & \text { Conclusions } & 9\end{array}$

\section{Introduction}

Gauge mediated supersymmetry breaking (GMSB) (for a review see [1]) is usually discussed in the context of global supersymmetry (SUSY) breaking. However when global SUSY is (spontaneously) broken a cosmological constant (CC) is generated at the same order as the supersymmetry breaking. It is only within the supergravity (SUGRA) context that one has the possibility of fine tuning the CC to zero. Thus any discussion of GMSB must necessarily be embedded within a supergravity context. It is then natural to ask whether such a SUGRA embedding can be justified/realized from string theory. In particular we would like to ask whether the small parameters in GMSB models (in effect mass scales which are highly suppressed relative to the Planck scale) can find a natural explanation in string theory. Within the global context such small mass scales have been justified by dynamical generation of a small scale in an asymptotically free gauge theory (in the socalled retrofitted models [2]). However this leaves the question of what fixes the relevant gauge coupling at the high scale, open. In string theory the gauge coupling (just like all other parameters) is a modulus which needs to be fixed by the dynamics of the model. Also typically the high scale in these models is necessarily well below the Planck scale. This too needs to be identified (in the string theory context) as either the mass of the lightest Kaluza-Klein state or the lightest modulus. In this note we explore these possibilities.

There is a large variety of GMSB models in the literature. What we will do here is to consider the simplest set up which exhibits all the essential features of these models. The issues that we are interested in here will be common to all GMSB models so there is no loss of generality in focusing on this simple set up. As we said before most discussions 
of these models are in the context of global SUSY. The embedding of such models within SUGRA was fist discussed by Kitano [3] who took the Kähler potential ${ }^{1}$

$$
K=X \bar{X}-\frac{(X \bar{X})^{2}}{\Lambda^{2}}+f \bar{f}+\tilde{f} \overline{\tilde{f}}+K_{\mathrm{MSSM}},
$$

and the superpotential

$$
W=c+\mu^{2} X+\lambda X f \tilde{f}+W_{\mathrm{MSSM}} .
$$

The non-renormalizable second term in $K$ arises from integrating out states at some high scale $\Lambda . X$ is a gauge neutral chiral superfield which is responsible for SUSY breaking. The superfields $f, \tilde{f}$ are to be identified as the messengers of GMSB and are charged under the gauge group. The model has a true minimum (i.e. with no tachyons or flat directions) with the fields taking values $X_{0}=\frac{\sqrt{3} \Lambda^{2}}{6}, f=\tilde{f}=0$, provided that $\Lambda^{4}>\frac{12 \mu^{2}}{\lambda}$ and the CC is tuned to zero i.e.

$$
\mu^{2} \simeq \sqrt{3} c=\sqrt{3} m_{3 / 2}
$$

Using the standard mass formula for scalar masses in SUGRA the mass matrices may be evaluated. The scalar messengers have squared masses

$$
\frac{\lambda^{2} \Lambda^{4}}{12} \pm \lambda \mu^{2}
$$

while the scalar partner of the Goldstino (sGoldstino) has a mass $m_{X} \simeq 2 \mu^{2} / \Lambda$ [4]. Finally, the SUSY breaking is characterized by

$$
F^{X} \simeq \mu^{2}=\sqrt{3} m_{3 / 2},
$$

so that the relevant mass parameter determining soft terms in GMSB is (restoring $M_{P}$ for clarity)

$$
m \sim \frac{\alpha}{4 \pi} \frac{F^{X}}{X} \simeq \frac{\alpha}{4 \pi} \frac{M_{P}^{2}}{\Lambda^{2}} 6 m_{3 / 2}
$$

This simple model illustrates the general requirements of GMSB. Note that gravity mediated SUSY breaking is always present and gives $m \sim m_{3 / 2}$. So in order to have GMSB dominance we need to enhance its contribution relative to the the gravity mediated one i.e.

$$
\frac{\alpha}{4 \pi} \frac{M_{p}^{2}}{\Lambda^{2}} \gg 1
$$

However we need to take $\Lambda$ to be even smaller if the corresponding gravity mediated model has FCNC. Thus we probably need the r.h.s. of the above inequality to be a factor of $10^{2}$ or so. Thus we need (assuming $\alpha / 4 \pi \sim 10^{-2}$ ), $\Lambda<10^{-2} M_{P}$. In fact, additional phenomenological constraints (which are usually imposed in the GMSB literature) suggest that we need to take a value which is much smaller.

Let us discuss these phenomenological constraints. We need $m \sim 100 \mathrm{GeV}-1 \mathrm{TeV}$, in order to 'solve' the hierarchy problem. The above considerations would then suggest that we could have a value of $m_{3 / 2} \sim 1 \mathrm{GeV}$. Such light gravitini were recognized long ago as

\footnotetext{
${ }^{1}$ We put $M_{P}=1$ in this work unless otherwise indicated.
} 
a source of problems in standard cosmology, see [5] for a review. However, it has been recognized by now that a wide range of gravitino masses between a few eV and 1-10 GeV can be tolerated depending on the mass of the NLSP, on the reheating temperature and on nonstandard events in the history of the universe, see for instance [6], [7], [8]. In the rest of the paper we will use $m_{3 / 2} \lesssim 1 \mathrm{keV}$ as a ball park figure. This implies

$$
\Lambda \lesssim 10^{-5} M_{P}
$$

For comparison we will also include (where relevant) the estimates for two other values for the gravitino mass: $m_{3 / 2} \sim 1 G e V$, corresponding to the case $\Lambda \sim 10^{-2} M_{P}$ mentioned above, and $m_{3 / 2} \sim 1 \mathrm{eV}$ (leading to $\Lambda \sim 10^{-7} M_{P}$ ). As it turns out, the main conclusion doesn't depend on a particular value of $m_{3 / 2}$, only numerical details do change.

In more general models of the hidden sector the situation can be even more severe. As observed in [9],

$$
m_{\mathrm{soft}}=\frac{\alpha}{4 \pi} \frac{F^{X}}{\Lambda^{\gamma}}
$$

where $\gamma=2$ for the Kitano model (order four correction to the Kähler potential balanced by a gravitational correction), $\gamma=1$ if R-symmetry becomes spontaneously broken due to radiative corrections in a globally supersymmetric model and $\gamma=4 / 3$ if the R-symmetry breaking is caused by an order six correction to a Kähler potential balanced by a gravitational correction. Assuming a $1 \mathrm{keV}$ gravitino, $\gamma=1$ results in $\Lambda \lesssim 10^{-10} M_{P}$ and $\gamma=4 / 3$ in $\Lambda \lesssim 10^{-7.5} M_{P}$. For the sake of concretness we are going to assume the conservative value $\gamma=2$.

The question we will address in the rest of the paper is, can we integrate out in a supersymmetric way all string theory moduli, to produce an effective theory with the scale of the lightest particle that is integrated out having a mass $\Lambda$ which is several orders of magnitude below the Planck scale. The essential point here is that the supersymmetry breaking mechanisms postulated in connnection with GMSB, are essentially four dimensional effective theories, which in a string theory context, are effectively obtained after decoupling all the string theory moduli, which therefore need to have been stabilized with their masses at some high scale. This is the only viable interpretation of the low energy supersymmetry breaking models that have been considered in the context of GMSB, when the question of their relation to string theory is considered. Otherwise one would have to look at the full effective theory coming from string theory, treating the moduli along with the fields of the low energy theory as dynamical variables, and this would certainly vitiate the arguments made in the context of the low energy supersymmetry breaking models. Thus the necessary criterion for embedding these models within string theory, is that the highest scale of the effective theory (i.e. $\Lambda$ ) should be lower than the mass of the lightest string theory modulus. We stress here that this requirement does not come from a cosmological (or phenomenological) constraint but from the need to construct an effective low energy theory from which all the moduli of string theory have been integrated out. 


\section{KKLT model}

The simplest possibility is to integrate out the moduli/dilaton in type IIB in a KKLT [10] like situation i.e. with a SUSY AdS minimum, and then tune the model of the previous section to uplift the CC. In particular this means that the gravitino mass (CC) at the KKLT stage should be comparable to the final gravitino mass i.e. $m_{3 / 2} \sim 10^{-24}$.

$$
\begin{aligned}
K & =-3 \ln (T+\bar{T})-k(U, \bar{U})-\ln (S+\bar{S}), \\
W & =A(U)+S B(U)+C e^{-a T},
\end{aligned}
$$

with $U=\left\{U^{a}, a=1, \ldots, h_{12}\right\}$. The first two terms in $W$ are generated by internal fluxes while the third is a non-perturbative (NP) contribution. The KKLT minimum preserves SUSY - all the moduli are determined by the equations

$$
D_{S} W=D_{a} W=D_{T} W=0 .
$$

Denoting by $W_{\text {flux }}$ the value of the first two terms in (2.2) we have, from the last equation above

and

$$
a C e^{-a T}=\frac{W_{\text {flux }}}{T+\bar{T}}
$$

$$
m_{3 / 2} \sim \frac{W_{0}}{(T+\bar{T})^{3 / 2}} \sim(T+\bar{T})^{-1 / 2} a C e^{-a T} .
$$

In these equations $T$ is the value of this modulus at the minimum.

As we pointed out before, in order to avoid additional fine tuning, we must take this to be at least as small as the final gravitino mass after uplift and SUSY breaking from the Polonyi type models of the previous section - i.e. we need the l.h.s. of the above relation to be $\sim 10^{-24}$.

On the other hand the mass of the Kähler modulus is

$$
m_{T} \sim \sqrt{T} a C e^{-a T},
$$

so that

$$
\frac{m_{T}}{m_{3 / 2}} \sim T
$$

The minimal requirement for getting Kitano models from this string construction is to have $m_{T} \gtrsim \Lambda \sim 10^{-5}$. Given the phenomenological bound $m_{3 / 2}<10^{-24}$, this gives a value $T \gtrsim 10^{19}$. However this would give a string scale (note that the volume of the CY is $\left.\sim 1 / T^{3 / 2}\right) m_{\text {string }} \sim M_{P} / \sqrt{T^{3 / 2}} \sim 10^{-14} M_{P}$. But this is much less than $\Lambda$ so that the model is inconsistent. In general the condition $m_{T} \gtrsim \Lambda$ leads to

$$
T>10^{19}\left(\frac{m}{100 \mathrm{GeV}} \frac{m_{3 / 2}}{1 \mathrm{keV}}\right)^{-1 / 2},
$$

hence raising $m$ and $m_{3 / 2}$ helps only slightly. Note that even with $m_{3 / 2} \sim 1 \mathrm{GeV}=$ $10^{-18} M_{P}$ we would get $m_{T} \gtrsim \Lambda \sim 10^{-2}$ (see para after (1.7)) which will give $T \sim 10^{16}$ 
and hence $m_{\text {string }} \sim 10^{-12} \ll \Lambda$. With a very light gravitino on other hand $\left(m_{3 / 2} \sim 1 \mathrm{eV}\right.$, $\left.\Lambda \sim 10^{-7} M_{P}\right)$ the above calculation gives $T \sim 10^{20}$ and $m_{\text {string }} \sim 10^{-15} M_{P} \ll \Lambda$. Clearly the model is inconsistent for any acceptable value for $m_{3 / 2}$ in GMSB.

\section{KKLT racetrack model in IIB}

The situation is considerably improved when we consider racetrack models within the KKLT context. The model is defined by

$$
\begin{aligned}
K & =-3 \ln (T+\bar{T})-k(U, \bar{U})-\ln (S+\bar{S}), \\
W & =A(U)+S B(U)+\sum C_{i} e^{-a_{i} T},
\end{aligned}
$$

with $U=\left\{U^{a}, a=1, \ldots, h_{12}\right\}$. Assume $C_{i}$ are constants i.e. ignore threshold effects. Obviously we need at least two NP terms. For condensing $\mathrm{SU}(N)$ groups $a_{i}=2 \pi / N_{i}$.

Let us look for SUSY Minkowski minima: $\partial W=W=0$,

$$
\begin{aligned}
& \partial_{S} W=B(U)=0, \partial_{T} W=-\sum_{i} a_{i} C_{i} e^{-a_{i} T}=0, \\
& \partial_{a} W=A_{a}(U)+B_{a}(U) S=0 .
\end{aligned}
$$

First and last equations are $h_{21}+1$ relations determining $U^{a}=U_{0}^{a}, S=S_{0}$. In the simplest case where we have just two NP terms the second equation gives

$$
e^{2 \pi \frac{N_{2}-N_{1}}{N_{1} N_{2}} T_{0}}=-\frac{N_{2}}{N_{1}} \frac{C_{1}}{C_{2}}
$$

With any solution of these equations for the moduli/dilaton, fluxes may be chosen such that $W\left(T_{0}, U_{0}, S_{0}\right)=0$.

Given a large number of 3-cycles, and the large number of choices of fluxes through them, one expects that the fine tuning of $W$ to zero is compatible with having the masses of the $U^{a}$, moduli and of axio-dilaton $S$ being very large - close to the string scale say. The lightest modulus would as usual be $T$ since it gets its mass from the NP terms,

$$
\left.m_{T}^{2} K_{T \bar{T}}\right|_{0}=\left.e^{K}\left|\partial_{T}^{2} W\right|^{2} K^{T \bar{T}}\right|_{0} M_{P}^{2} \sim \frac{1}{T_{0}+\bar{T}_{0}}\left|M_{P}^{2} \sum_{i}\left(\frac{2 \pi}{N}\right)^{2} e^{-2 \pi T_{0} / N}\right|^{2} .
$$

In the last relation we have estimated the size of the Kähler potential terms from $U, S$ to be of $O(1)$. Note that $K_{T \bar{T}}=3 /(T+\bar{T})^{2}$. The following results are simple consequences of this set up:

- The KK scale is $M_{K K} \sim M_{P} / \Re T_{0}$ and the consistency of the whole framework requires that this mass be greater than $m_{T}$. This implies that

$$
m_{T} \sim\left(T_{0}+\bar{T}_{0}\right)^{1 / 2}\left|\sum_{i}\left(\frac{2 \pi}{N_{i}}\right)^{2} e^{-2 \pi T_{0} / N_{i}}\right| M_{P}<\frac{M_{P}}{\left(T_{0}+\bar{T}_{0}\right)} .
$$

Now the phenomenology of GMSB requires (1.7). If we identify $\Lambda$ with the KK mass this requires $T_{0} \gtrsim 10^{5}$. 
- If we identify $\Lambda$ with the lower mass $m_{T}$ clearly we need a value of $T$ which is parametrically larger than the Planck scale. This implies that we need large values of $N_{1}, N_{2}$ so that from (3.5) we have (taking $N_{2}>N_{1}$ )

$$
\tau_{0} \sim \frac{N_{2}}{2 \pi}
$$

where $\tau_{0}$ is the real part of $T$ at the minimum. This gives

$$
m_{T} \sim\left(2 \pi / N_{2}\right)^{3 / 2} M_{P} \sim M_{P} / \tau_{0}^{3 / 2} .
$$

Identifying this with $\Lambda \lesssim 10^{-5} M_{P}$ gives, $\tau_{0} \gtrsim 3 \times 10^{3}$. This however requires very large gauge groups

$$
N_{1}, N_{2} \gtrsim 10^{4}
$$

These are rather large values, but in certain F-theory constructions [11] such large groups have in fact been obtained, though it is not clear to us how common they are in the landscape of IIB orientifold compactifications (or the corresponding F-theory constructions).

Note that the estimate in (3.8) and hence (3.10) were made under the assumption that any difference in the ratio of the pre-factors $C_{1} / C_{2}$ from unity should disappear for large ranks, i.e. $\left|\frac{C_{1}}{C_{2}}\right|-1 \lesssim O(1 / N)$ since the only difference between them comes from the $O(1)$ difference between the ranks. The explicit dependence on the rank (which is governed by the number of D7 branes wrapping the four cycle) calculated in [12] certainly satisfies this. If however for some reason there are large threshold effects which make $C_{1} / C_{2}$ significantly larger than 1 then these estimates would be changed. In this case we would have $\tau_{0} \sim \frac{N^{2}}{2 \pi}$, so that $N_{1}, N_{2} \sim O(10)$. It is an interesting problem to propose a source of such large threshold corrections in the string theoretic context.

\section{LVS models}

A natural way of getting a large volume (which seems to be needed to explain $\Lambda \lesssim 10^{-5} M_{P}$ ) is to consider LVS models [13]. These need at least two Kähler moduli with one of them being a blow-up modulus. The simplest example has the CY volume being of the form

$$
\mathcal{V}=\tau_{b}^{3 / 2}-\tau_{s}^{3 / 2}
$$

Here $\tau_{b, s}$ govern the size of the large 4-cycle and the blown-up four cycle respectively. When the potential in minimized we get

$$
\begin{aligned}
e^{-a \tau_{s}} & \simeq \frac{3}{4} \frac{W_{0}}{a A \mathcal{V}} \sqrt{\tau_{s}}\left(1-\frac{3}{4 a \tau_{s}}\right), \\
\tau_{s}^{3 / 2} & \simeq \frac{\hat{\xi}}{2}\left(1+\frac{1}{2 a \tau_{s}}\right) .
\end{aligned}
$$

Here

$$
\hat{\xi}=-\frac{1}{g_{\text {string }}^{3 / 2}} \chi \zeta(3) / 2(2 \pi)^{3}
$$


where $\chi$ is the Euler character of the manifold and $\zeta$ is the Riemann zeta function. Noting that $m_{3 / 2} \sim W / \mathcal{V}$ the first equation gives

$$
a \tau_{s} \sim\left|\ln m_{3 / 2}\right|
$$

The LVS solution breaks SUSY, hence in order to be compatible with GMSB we have to tune the gravitino mass to be at or below the GMSB favored value $m_{3 / 2} \lesssim 1 \mathrm{KeV}=$ $10^{-24} M_{P}$. So from (4.4) we need $a \tau_{s} \sim 10^{2}$ which implies (given that $a \sim O(2 \pi)$ at most) from (4.2) that $\hat{\xi} \sim O(10)$ and hence from (4.3) we need CY orientifolds of large Euler character $(\chi \sim 5000)$. While there are no known barriers to having such large Euler characters, the largest found so far have values that are around an order of magnitude smaller. Of course given the logarithmic dependence on $m_{3 / 2}$ the above conclusion will remain true for the whole range of values for this mass discussed in the introduction.

In any case in this type of scenario the light modulus has a mass $m \sim m_{3 / 2} / \sqrt{\mathcal{V}}$ which is obviously below the messenger scale and hence cannot be integrated out to give the type of action (like the Kitano model) that GMSB needs.

\section{$5 \quad$ Retrofitted models}

The model of supersymmetry breaking in (1.1)(1.2) has three parameters that are highly suppressed relative to the Planck scale. In the previous section we identified $\Lambda \sim 10^{-5}$ as the scale set by the lowest modulus mass which is integrated out. The other two parameters $c, \mu^{2}$ have to be fine tuned against each other to get zero CC after SUSY breaking, and they have to be extremely tiny in order to get a $1 \mathrm{keV}$ (or even $1 \mathrm{GeV}$ ) gravitino mass. This means that we need

$$
\mu^{2} \simeq \sqrt{3} c=\sqrt{3} m_{3 / 2} \sim O\left(10^{-24}\right)
$$

How does such a small parameter arise. In retrofitted models [2] this is related to a dynamically generated scale as in QCD. In the context of SUSY QCD this may be identified with gaugino condensation. So we effectively have

$$
\mu^{2}=\Sigma^{3}=\Lambda^{3} \exp \left(-\frac{3}{b} \frac{8 \pi^{2}}{g^{2}(\Lambda)}\right)
$$

Here $\Sigma$ is the dynamically generated scale, $\Lambda$ is the UV scale of the effective theory that we identified in the previous section, $g(\Lambda)$ is the coupling at that scale of some gauge group, and $b$ is the corresponding one-loop beta-function coefficient (for example $b=3 N$ for pure $\mathrm{SU}(N)$ Yang-Mills). Even for very small gauge groups with say $N=2$ this requires a gauge coupling constant at the UV scale to be quite small $\alpha=\frac{g^{2}}{4 \pi} \sim 0.06$ to satisfy the estimate (5.1).

In string theory however this coupling constant is actually a field. As we discussed in the previous section it is most naturally identified with the modulus $T$. Then we might try to identify the supersymmetrty breaking sector (Polonyi field) with the open string moduli (for simplicity represented by a single field $X$ here), that describe the location of 
the stack of D3 branes relative to the stack of D7 branes which wrap the four cycle in the CY space, and generate the NP term in (3.2). Following Baumann et al [12] (in particular its adaptation to our situation in [14]) we should modify this term to read

$$
\sum A\left(1-\frac{X}{\sigma}\right)^{1 / N_{i}} e^{-\frac{2 \pi}{N_{i}} T}
$$

where $\sigma$ represents the location of the $\mathrm{D} 7$ stack in the internal space. The natural scale at which to make the identification the guage coupling $\alpha=T$, would be the Planck scale. In this case clearly we cannot produce the small number that is necessary to satisfy (5.1) since the exponential factor is fixed by the racetrack to be $\exp \{-O(1)\}$. Even if this identification is made at the cut off scale $\Lambda$, where we would have $\alpha(\Lambda)=T$ and $A=\Lambda^{3}$, the exponential factor would still need to produce a factor of around $10^{-9}$ relative to $\Lambda^{3}$ (see (5.2)) to get a gravitino at the $\mathrm{keV}$ scale. This is clearly not possible.

The alternative is to couple $X$ to the theory on a stack of D3 branes. Perhaps this field can be considered as a modulus describing the location of this stack relative to the standard model stack which is located at some singularity. Assuming a condensing gauge group on the stack of D3 branes, and that the corresponding gauge coupling at some high scale, which we again take to be $\Lambda$, is $S$ the dilaton, we have $\alpha(\Lambda)=S$. Unlike in the case discussed above we do not know of a string construction that accomplishes this. Nevertheless let us ask whether a term such as

$$
\Lambda^{3} X e^{-\frac{2 \pi}{N} S}
$$

can account for the $\mu^{2} X$ term in the Polonyi model. To get the appropriate suppression (with $\Lambda=10^{-5}$ ) seems to require $S=\frac{\partial_{U} A}{\partial_{U} B} \sim 5-10$ with low values of $N$. On the other hand the mass of the dilaton is given by

$$
m_{S} \sim \sqrt{s} \frac{\left|\partial_{U} B\right|}{\tau_{0}^{3 / 2}}
$$

This is to be compared with the mass $m_{T} \sim 1 / \tau_{0}^{3 / 2}$ (see (3.9)). Clearly fluxes can be chosen such that the masses satisfy the consistency condition $m_{S}>m_{T} \sim \Lambda$. If on the other hand (as is more natural in SUGRA) we identified the high scale at which the boundary value of the coupling is equal to $S$ as the Planck scale (so effectively replacing the pre-factor $\Lambda^{3}$ by 1 in (5.4)) we would need (with low values of $N$ ) values of $S$ of $O(10)$. Again there does not appear to be any problem with this.

\section{Models with charged moduli - role of D-terms}

Finally let us consider a scenario where all but the Kähler modulus have been integrated out close to the string scale. In this case an interesting class of models corresponds to the situation, when this modulus is charged under an anomalous $\mathrm{U}(1)$ gauge group (equivalently one can say that the shift of the modulus has been gauged). Then the expectation is that the modulus becomes a component of the massive vector multiplet and, indeed, decouples 
from the light spectrum, see [15]. This question has been studied for example in the last reference, and more recently in [16] (in the context of Type I string theory, with a D1instanton inducing the Polonyi term) and in [17]. To be more specific, let us consider the superpotential

$$
W=W_{0}+f X e^{-T},
$$

invariant under an U(1). For the sake of discussion let us first consider the Kähler potential

$$
K=|X|^{2}-\frac{|X|^{4}}{\Lambda^{2}}+\frac{m_{V}^{2}}{2}\left(T+T^{\dagger}-V\right)^{2},
$$

where $m_{V}$ is the mass of the gauge boson of the $\mathrm{U}(1)$. In addition to the F-term scalar potential one finds also a non-trivial D-term contribution of the form

$$
V_{D}=\frac{1}{2}\left(\left(|X|^{2}-2 \frac{|X|^{4}}{\Lambda^{2}}\right)+m_{V}^{2}\left(T+T^{\dagger}\right)\right)^{2} .
$$

One finds that the expectation value of $X$ is fixed as in the Kitano model and the D-term fixes the expectation value of the modulus at $\langle T\rangle=\frac{\Lambda^{4}}{24 m_{V}^{2}\left(1+3 \frac{\Lambda^{2}}{m_{V}^{2}}\right)^{2}}[17]$. The mass of the real part of $T$ is $2 m_{V}$ while the imaginary part of the modulus is swallowed by the massive gauge boson. Taking $m_{V}$ of the order of the compactification scale one concludes that the modulus decouples. However, the resulting expectation value of the modulus depends crucially on the form of its Kähler potential. In the above example we have assumed a quadratic Kähler potential for the $\mathrm{T}$, which implies a small expectation value and an unsuppressed effective mass scale multiplying $X$ in the Polonyi term. Unfortunately, for the Kähler modulus the appropriate form is logarithmic, $K=-3 \log \left(T+T^{\dagger}\right)$. This in turn gives $\sim \frac{3}{T+T^{\dagger}}$ in the D-term, which implies a run-away vacuum solution for $T$, since the term in $V_{D}$ which depends on $X$ must be very small. Large values of $\langle T\rangle$ suppress very strongly the effective mass scale in the Polonyi term making the model unrealistic (see also [18]).

\section{Conclusions}

We have found that (within the well understood context of IIB flux compactifications), simple models which allow for GMSB like scenario below the lowest modulus mass scale are possible, but they are rather unlikely in the context of the landscape. In LVS type compactifications [13] the string theory sector cannot be integrated out since there is a light modulus that is below the messenger scale. The simplest KKLT type model (i.e. with just one non-perturbative exponential term) will not work since when the necessary constraints for realizing a GMSB scenario are imposed, we get a string scale that is below the messenger scale and so is inconsistent. The only possibility (as far as we can see) is to have an extended KKLT scenario, with more than one NP term, i.e. a racetrack. In this case we can get the necessary conditions, however the resulting gauge groups are anomalously large, though it may be possible to obtain them in some F-theory constructions. Even if that were the case, the simplest possibility for explaining the low value of the Polonyi scale (i.e. the SUSY breaking scale) does not work, and while we have suggested an alternative it is not 
clear that this can actually be realized in string theory. Finally we made some comments about the possibility of D-term effects and concluded that they will not help if the Kähler potential for the relevant modulus is of the form that one usually gets in string theory.

\section{Acknowledgments}

The research of SdA is partially supported by the United States Department of Energy under grant DE-FG02-91-ER-40672. The research of ZA is partially supported by Polish Ministry for Science and Education under grant N N202 091839. Both of us wish to thank KITP, Santa Barbara for hospitality while this work was in its formative stages.

Open Access. This article is distributed under the terms of the Creative Commons Attribution Noncommercial License which permits any noncommercial use, distribution, and reproduction in any medium, provided the original author(s) and source are credited.

\section{References}

[1] G.F. Giudice and R. Rattazzi, Theories with gauge-mediated supersymmetry breaking, Phys. Rept. 322 (1999) 419 [hep-ph/9801271] [SPIRES].

[2] M. Dine, J.L. Feng and E. Silverstein, Retrofitting O'Raifeartaigh models with dynamical scales, Phys. Rev. D 74 (2006) 095012 [hep-th/0608159] [SPIRES].

[3] R. Kitano, Gravitational gauge mediation, Phys. Lett. B 641 (2006) 203 [hep-ph/0607090] [SPIRES].

[4] S.P. de Alwis, A Comparison of Supersymmetry Breaking and Mediation Mechanisms, arXiv: 1006.0949 [SPIRES].

[5] M. Drees, R. Godbole and P. Roy, Theory and phenomenology of sparticles: An account of four-dimensional $N=1$ supersymmetry in high energy physics, World Scientific, Singapore (2004).

[6] J.L. Feng, M. Kamionkowski and S.K. Lee, Light Gravitinos at Colliders and Implications for Cosmology, Phys. Rev. D 82 (2010) 015012 [arXiv: 1004.4213] [SPIRES].

[7] M. Bolz, A. Brandenburg and W. Buchmüller, Thermal Production of Gravitinos, Nucl. Phys. B 606 (2001) 518 [hep-ph/0012052] [SPIRES].

[8] L. Covi, M. Olechowski, S. Pokorski, K. Turzynski and J.D. Wells, Supersymmetric mass spectra for gravitino dark matter with a high reheating temperature, JHEP 01 (2011) 033 [arXiv: 1009.3801] [SPIRES].

[9] Z. Lalak, S. Pokorski and K. Turzynski, Gravity in Gauge Mediation, JHEP 10 (2008) 016 [arXiv:0808.0470] [SPIRES].

[10] S. Kachru, R. Kallosh, A.D. Linde and S.P. Trivedi, de Sitter vacua in string theory, Phys. Rev. D 68 (2003) 046005 [hep-th/0301240] [SPIRES].

[11] P. Candelas, E. Perevalov and G. Rajesh, Toric geometry and enhanced gauge symmetry of F- theory/heterotic vacua, Nucl. Phys. B 507 (1997) 445 [hep-th/9704097] [SPIRES].

[12] D. Baumann et al., On D3-brane potentials in compactifications with fluxes and wrapped D-branes, JHEP 11 (2006) 031 [hep-th/0607050] [SPIRES]. 
[13] V. Balasubramanian, P. Berglund, J.P. Conlon and F. Quevedo, Systematics of Moduli Stabilisation in Calabi-Yau Flux Compactifications, JHEP 03 (2005) 007 [hep-th/0502058] [SPIRES].

[14] S.P. de Alwis, On gauge mediated SUSY breaking and moduli stabilization, Phys. Rev. D 76 (2007) 086001 [hep-th/0703247] [SPIRES].

[15] Z. Lalak, S. Lavignac and H.P. Nilles, String dualities in the presence of anomalous U(1) symmetries, Nucl. Phys. B 559 (1999) 48 [hep-th/9903160] [SPIRES].

[16] M. Cvetič and T. Weigand, A string theoretic model of gauge mediated supersymmetry beaking, arXiv:0807.3953 [SPIRES].

[17] T. Jelinski, Z. Lalak and J. Pawełczyk, Non-minimal Gauge Mediation and Moduli Stabilization, Phys. Lett. B 689 (2010) 186 [arXiv:0912.3735] [SPIRES].

[18] E. Dudas, Y. Mambrini, S. Pokorski, A. Romagnoni and M. Trapletti, Gauge vs. Gravity mediation in models with anomalous $U(1)$ 's, JHEP 03 (2009) 011 [arXiv:0809.5064] [SPIRES]. 